\title{
Formulación lineal de extensiones del problema de localización competitiva del líder- seguidor
}

\author{
CLARA M. CAMPOS RODRÍGUEZ \\ Instituto Universitario de Desarrollo Regional, UNIVERSIDAD DE LA LAGUNA, \\ ESPAÑA.E-mail: ccampos@ull.es
}

DOLORES R. SANTOS PEÑATE

Departamento de Métodos Cuantitativos en Economía y Gestión, UNIVERSIDAD DE LAS PALMAS DE G.C., ESPAÑA. E-mail: drsantos@dmc.ulpgc.es

JOSÉ ANDRÉS MORENO PÉREZ

Instituto Universitario de Desarrollo Regional, UNIVERSIDAD DE LA LAGUNA, ESPAÑA.E-mail: jamoreno@ull.es

\section{RESUMEN}

El problema del líder-seguidor es un problema de decisión secuencial en un mercado sin centros de servicio, donde una empresa, la empresa líder, abre primero sus centros y otra empresa competidora, la empresa seguidora, entra más tarde en el mercado abriendo los suyos. La empresa líder elige las localizaciones teniendo en cuenta que la empresa seguidora abrirá sus instalaciones y capturará parte de la cuota de mercado. El objetivo de cada una de las firmas competidoras es maximizar su cuota de mercado. Consideramos que el consumidor se comporta haciendo una elección binaria utilizando la distancia como criterio de elección. Extendemos el modelo básico contemplando un umbral en la regla de elección del cliente y costes de apertura dependientes de la localización. Formulamos las extensiones del problema como un programa lineal a partir del modelo lineal básico.

Palabras clave: Localización competitiva, centroide, problema del líder-seguidor, problema de Stackelberg.

\section{Linear Formulation for Extensions of the Leader-Follower Competitive Location Problem}

\begin{abstract}
The leader-follower problem is a sequential decision problem where, in a market initially without service centers, a firm, the leader, opens its centers and another competing firm, the follower, will enter later the market opening its centers. The leader chooses the locations taking into account that the follower will enter the market opening its centers and capturing part of the market share. The objective of each competing firm is to maximize its market share. We consider that the clients will behave making a binary choice using the distance as basis for her/his choice criterion. We extend the basic model by considering a threshold in the client choice rule and opening costs depending on the location. We get formulations of the problem extensions as linear programs from the basic linear model.
\end{abstract}

Keywords: Competitive Location, Centroid, Leader-Follower Problem, Stackelberg Problem.

Clasificación JEL: C69

\footnotetext{
* Este trabajo ha sido parcialmente financiado por el Gobierno de España y FEDER (Referencias ECO2008-05589 y TIN2008-06872-C04-01).
}

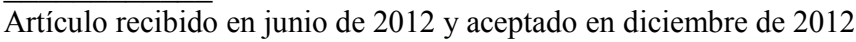

Artículo disponible en versión electrónica en la página www.revista-eea.net, ref. ə-31105 


\section{INTRODUCCIÓN}

Los modelos de localización competitiva representan situaciones en las que dos o más empresas compiten para alcanzar ciertos objetivos. Normalmente el objetivo es maximizar los beneficios o la cuota de mercado, aunque pueden perseguirse otros como la minimización de la cuota de mercado del competidor o la maximización de la diferencia entre la cuota de mercado propia y la del competidor. Cuando los bienes ofrecidos por las empresas son esenciales la demanda debe satisfacerse en su totalidad y se reparte entre las firmas que operan en el mercado, en este caso los tres objetivos mencionados son equivalentes.

Obviamente, la variable de decisión relevante en un modelo de localización es la ubicación de los centros de servicio, sin embargo el problema puede incorporar otras, por ejemplo el precio del producto, la cantidad ofertada, el tamaño de los centros, y otros atributos de las instalaciones. En este trabajo consideramos que la única variable de decisión es la localización.

En general, la decisión del consumidor suele basarse en la distancia o tiempo de desplazamiento hasta los centros de servicio y en algunas propiedades de estos centros, todo ello conforma el atractivo de los centros para los clientes. Por otro lado, el comportamiento del cliente viene modelado por la regla de elección del consumidor, dicha regla determina los centros que serán visitados por los clientes. Algunas de las reglas de decisión que podemos encontrar en la literatura son las siguientes:

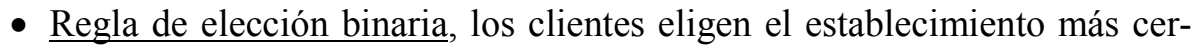
cano.

- Regla de elección parcialmente binaria, los clientes eligen el establecimiento más cercano de cada una de las firmas que operan en el mercado.

- Regla de elección proporcional, los clientes eligen todos los establecimientos y utilizan en ellos una cantidad de poder de compra que viene dada por una función decreciente de la distancia desde el cliente al establecimiento.

- Reglas de elección gradual, donde la proporción de demanda en un punto capturada por un centro viene dada por una función decreciente de la distancia entre dicho punto y el centro. Si esta distancia supera cierto valor entonces la función toma el valor 0 , y si es inferior a otro valor dado entonces el valor de la función es 1 .

Aunque es poco realista pensar que pequeñas diferencias en la distancia son perceptibles para el individuo, el modelo binario es útil para las aplicaciones en las que el producto puede ser considerado homogéneo y se supone que los establecimientos son idénticos, como por ejemplo los quioscos de periódicos y las farmacias. Otros modelos sustituyen esta regla de elección por otras que incorporan un umbral de sensibilidad y funciones de reparto, de manera que, dentro 
de cierto rango de la distancia al establecimiento, la demanda se reparte entre las firmas competidoras (ver, por ejemplo, Devletoglou (1965), Devletoglou y Demetriou (1967) y Berman y Krass (2002)). Opuesta a la regla binaria se encuentra la regla de elección proporcional (Hakimi, (1990), según esta regla un cliente utiliza todos los establecimientos visitándolos de acuerdo a una función decreciente de la distancia que lo separa de ellos. En este caso, el modelo incorpora una función dependiente de las distancias entre clientes y establecimientos que da la proporción de demanda en un punto captada por un centro, esta proporción puede interpretarse como la probabilidad de que un cliente acuda a un establecimiento en función de sus localizaciones.

Otro elemento de los modelos de localización competitiva es el estado del mercado o escenario donde las firmas van a ejecutar sus decisiones. Podemos considerar las situaciones siguientes:

- No existen firmas operando en el mercado y la firma $F_{1}$ quiere entrar en el mercado con $p_{1}$ establecimientos.

- Ya hay $s$ firmas operando en el mercado. Cada firma $F_{i}$ tiene $p_{i}$ establecimientos $i=1, \ldots, s, \mathrm{y}$ una nueva firma $F_{s+1}$ quiere entrar en el mercado con $p_{s+1}$ establecimientos.

- Hay $s$ firmas operando en el mercado. Cada firma $F_{i}$ tiene $p_{i}$ establecimientos $i=1, \ldots, s$ y la firma $F_{k}$ quiere abrir $\bar{r}_{k}$ establecimientos y cerrar $\underline{r}_{k}$ establecimientos.

El problema de localización competitiva del líder-seguidor o problema de Stackelberg es un problema de decisión secuencial donde la empresa líder entra primero en el mercado teniendo en cuenta que en el futuro otra empresa, la empresa seguidora, entrará en el mercado situando sus centros en las localizaciones que le proporcionen la cuota de mercado máxima. Considerando que no existen empresas operando en el mercado, la firma líder, $F_{1}$, quiere abrir $p_{1}$ establecimientos en las localizaciones que minimicen la máxima cuota de mercado que los competidores futuros puedan alcanzar.

Una solución de Stackelberg o $(r \mid p)$-centroide es un par $\left(X_{p}^{*}, Y_{r}^{*}\right)$ donde $Y_{r}^{*}$ es la estrategia óptima del seguidor si el líder tiene $p$ establecimientos localizados en $X_{p}^{*}, \mathrm{y} X_{p}^{*}$ es la estrategia preventiva óptima del líder, suponiendo que el seguidor abre $r$ centros de servicio.

La denominación $(r \mid p)$-centroide para referirse a la estrategia óptima del líder fue introducida por Hakimi (1990) para el problema en redes. A la solución óptima del seguidor cuando el líder instala sus centros en el conjunto $X_{p}$ la llamó $\left(r \mid X_{p}\right)$-medianoide. La estructura de red es uno de los espacios posibles donde puede plantearse un problema de localización. En ese caso un centro de servicio puede abrirse en cualquier punto de la red y hablaremos de problema de localización en redes. El espacio donde se define un problema de localización 
puede también ser continuo, por ejemplo el plano, o discreto, en cuyo caso el conjunto de puntos donde pueden instalarse los centros es discreto, normalmente finito. Para los problemas en redes, algunos resultados sobre la existencia de solución óptima en el conjunto de vértices para el problema del $\left(r \mid X_{p}\right)$-medianoide en diferentes escenarios, pueden encontrarse en Hakimi (1964) y Suárez-Vega et al. (2004). Un estudio reciente de Spoerhase y Wirth (2007) incluye un resultado de la discretización para el (1|p)-centroide en un árbol. Los resultados de la discretización permiten la resolución de problemas en redes usando herramientas diseñadas para resolver problemas en espacios discretos. En ciertos escenarios, estos resultados garantizan la existencia de un $\left(r \mid X_{p}\right)$-medianoide en el conjunto de vértices de la red el cual se convierte en el conjunto de localizaciones candidatas para abrir los establecimientos. En otras situaciones, el conjunto de candidatos depende de las localizaciones elegidas por el líder, haciendo el problema más difícil.

Revisiones de los modelos de localización competitiva pueden encontrarse en Eiselt y Laporte (1989), Eiselt et al. (1993), Friesz et al. (1988), Plastria (2001), y Suárez-Vega et al. (2004), entre otros. En Santos-Peñate et al. (2007) se presenta un resumen del modelo líder-seguidor en redes. Kress y Pesch (2012) presentan una revisión de los modelos de localización competitiva secuencial en redes. ReVelle (1986) formuló el Problema de Captura Máxima, éste es el problema del seguidor discreto considerando la localización de varios establecimientos, con una regla de elección binaria y demanda esencial. Una revisión del Problema de Captura Máxima y algunas extensiones se presenta en Serra y ReVelle (1995). Algunos modelos incorporan, además de la localización, otras variables que representan el atractivo de los establecimientos, consideran otros objetivos como la maximización de una función de beneficios, o involucran problemas de equilibrio localización-precio, localización-cantidad o localización-atractivo (veáse, por ejemplo, Santos-Peñate et al. (1999)).

El problema de elección secuencial del líder-seguidor se formula como un proceso de optimización en tres niveles simultáneos. El proceso incluye:

- El problema de elección del cliente: dadas las localizaciones del líder y del seguidor, escoger el punto de servicio preferido.

- El problema de localización del seguidor: dadas las localizaciones del líder, seleccionar el conjunto de localizaciones del seguidor que maximiza la demanda total captada por éste.

- El problema de localización del líder: obtener el conjunto de localizaciones del líder que minimiza la máxima demanda que puede capturar el seguidor.

La resolución del problema del líder-seguidor presenta cierta dificultad ya que para cada posible opción del líder la respuesta del seguidor es la solución de 
un problema de optimización que es preciso resolver. No hay demasiadas propuestas de algoritmos de solución para este problema de localización secuencial. Entre los artículos que muestran procedimientos para encontrar una solución de Stackelberg tenemos los trabajos de Alekseeva et al.(2010), Benati y Laporte (1994), Bhadury et al. (2003), Campos-Rodríguez et al. (2010), Redondo et al. (2010), Serra y ReVelle (1994) y (1995), y Spoerhase y Wirth (2009).

En este trabajo consideramos el problema de localización del líder-seguidor discreto para bienes esenciales y una regla de elección binaria. La única variable de decisión es la localización y la decisión del consumidor se basa únicamente en la distancia de desplazamiento. La función objetivo es la cuota de mercado. En el modelo básico, el del $(r \mid p)$-centroide, se considera que la única limitación sobre las localizaciones de los competidores viene dada por el número de centros a establecer, entendiéndose que todas las localizaciones llevan asociado el mismo coste de apertura de un centro. Proponemos otras versiones del modelo, extendiendo el modelo básico mediante la introducción de un umbral para diferencia de distancias en la regla de elección binaria y de costes de instalación dependientes de la localización. Ambas extensiones obedecen a la necesidad de contemplar modelos más próximos a la realidad. El umbral permite reflejar el hecho de que los clientes deben percibir una diferencia significativa en las distancias para manifestar una preferencia que influya en su elección. Los costes variables permiten reflejar el hecho de que el coste de instalación de los centros depende de manera significativa de la localización elegida. En esta extensión una restricción presupuestaria sustituye a la limitación sobre el número de centros que puede abrir cada empresa. Ambas extensiones se pueden considerar de forma simultánea e incluyen al modelo básico como caso particular con umbral nulo y coste uniforme de las localizaciones.

El trabajo está organizado de la manera siguiente. En la sección 2 se describe el modelo básico del líder-seguidor. Su formulación como un programa lineal se incluye en la sección 3. En la sección 4 presentamos las extensiones del modelo básico y sus formulaciones como programas lineales. La sección 5 contiene un ejemplo. Finalmente, en la sección 6 incluimos algunas conclusiones.

\section{EL MODELO BÁSICO}

Sea $G(V, E)$ un grafo o red y $C, L \subseteq V$ conjuntos con cardinal igual a $|C|=n$ y $|L|=m$. $C$ es el conjunto de las localizaciones de los clientes, los puntos de demanda, y $L$ es el conjunto de posibles localizaciones de centros de servicio. Sea $d(c, x)$ la distancia desde la localización $c \in C$ a la localización $x \in L$. Sea $w$ una función de pesos sobre el conjunto de las localizaciones de los clientes, donde el peso $w(c)$ de la localización $c$ representa la demanda de los clientes ubicados en ese punto. 
Primero consideramos el modelo en el que cada competidor va a localizar un único establecimiento. Denotemos por $x$ a la localización del líder y por $y$ a la localización del seguidor. Para dos puntos $x$ e $y$ de $L$, la preferencia de cada cliente en $c$ se establece por la siguiente regla "el establecimiento en $y \in L$ es preferido por el cliente en $c$ a $x \in L$ si $d(c, y)<d(c, x)$ ". En el caso de empates, esto es, cuando las distancias del cliente al líder y al seguidor coinciden, esta regla favorece al líder ya que sería éste quien capturaría toda la demanda en el punto $c$.

Para cada par de localizaciones $x, y \in \mathrm{L}$, sea $C(y \prec x)$ el conjunto de localizaciones de clientes que cambian su elección de la localización $x$ por la localización $y$ más cercana. Entonces

$$
C(y \prec x)=\{c \in C: d(c, y)<d(c, x)\} .
$$

La demanda total de los clientes que cambiarían a la localización $y$ desde la localización $x$ se denota $W(y \prec x)$ y vale:

$$
W(y \prec x)=\sum_{c \in C(y \prec x)} w(c) .
$$

Sea

$$
W^{*}(x)=\max _{y \in L} W(y \prec x) .
$$

Un $x$-medianoide es un punto $y \in L$ tal que $W^{*}(x)=W(y \prec x)$. Un centroide es un punto $x^{*} \in L$ tal que

$$
W^{*}\left(x^{*}\right)=\min _{x \in L} W^{*}(x) .
$$

Un centroide es un punto de $L$ tal que la demanda total máxima de los clientes que prefieren otro punto de $L$ es la menor posible. Formalmente, un punto $x \in L$ es un centroide para el espacio de localizaciones $L$ y para el conjunto de puntos de demanda $C$ si minimiza su puntuación, es decir $x \in L$ es un centroide si

$$
\forall y \in L: \quad W^{*}(x)=\max _{z \in L} W(z \prec x) \leq W^{*}(y)=\max _{z \in L} W(z \prec y) .
$$

Así el problema del centroide es el problema minimax

$$
\min _{x \in L} \max _{z \in L} W(z \prec x) .
$$

El conjunto $X^{*}$ de centroides viene dado por:

$$
X^{*}=\arg \min _{x \in L} W^{*}(x)=\arg \min _{x \in L} \max _{z \in L} W(z \prec x) .
$$

Consideremos ahora el problema de localización múltiple en el que los puntos $x$ e $y$ de $L$ son sustituidos por conjuntos de $p$ y $r$ localizaciones, respectiva- 
mente. Esto es, sea $p$ el número de establecimientos que abrirá el líder y $r$ el número de establecimientos que instalará el seguidor.

Sea $L^{q}$ el conjunto de subconjuntos de $L$ con $q$ puntos, es decir

$$
L^{q}=\{X \subseteq L:|X|=q\} .
$$

La distancia desde la localización $c$ de un cliente hasta un conjunto de localizaciones $Z \subseteq L$ es

$$
d(c, Z)=\min \{d(c, z): z \in Z\} .
$$

Los clientes que cambian su elección al punto $y \in L$ desde un conjunto $X \subseteq L$ son aquellos más cercanos a y que a cualquier punto de $X$. Por lo tanto

$$
C(y \prec X)=\bigcap_{x \in X} C(y \prec x)=\{c \in C: d(c, y)<d(c, X)\} .
$$

El conjunto $C(Y \prec X)$ de localizaciones de clientes que captura el conjunto de localizaciones $Y \subseteq L$ frente al conjunto de localizaciones $X \subseteq L$ es el conjunto de puntos de $C$ para los que existe un punto de $Y$ más cerca que cualquier punto de $X$. Así

$$
C(Y \prec X)=\{c \in C: d(c, Y)<d(c, X)\} .
$$

Éste es el conjunto de localizaciones de puntos $c$ tales que para cualquier punto $x \in X$ existe un punto $y \in Y$ más cerca del cliente en $c$ de lo que lo está $x$, este punto $y$ depende del punto $c$ y de la localización $x$. El conjunto $C(Y \prec X)$ se puede obtener de la siguiente forma:

$$
\begin{aligned}
& C(Y \prec X)=\bigcup_{y \in Y} C(y \prec X)=\bigcup_{y \in Y} \bigcap_{x \in X} C(y \prec x)= \\
& =\{c \in C: \forall x \in X \exists y \in Y: d(c, y)<d(c, x)\} .
\end{aligned}
$$

Finalmente, la demanda total de los clientes que captura el conjunto $Y$ al conjunto $X$ es:

$$
W(Y \prec X)=\sum_{c \in C(Y \prec X)} w(c) .
$$

Estas definiciones permiten extender las nociones de solución dadas para el caso en que las firmas competidoras instalan un único centro al caso en que las firmas desean determinar las localizaciones para varios establecimientos.

Si $X \subseteq L$ es el conjunto de las localizaciones de los establecimientos del líder, entonces un $(r \mid X)$-medianoide es el mejor conjunto de $r$ puntos para abrir los centros del seguidor. Formalmente, un conjunto de localizaciones $Y \in L^{r}$ es un $(r \mid X)$-medianoide para el conjunto de puntos de demanda $C$ si y solamente si

$$
W(Y \prec X) \geq W(Z \prec X), \quad \forall Z \in L^{r} .
$$


Denotamos $Y_{r}(X)$ al conjunto de $(r \mid X)$-medianoides, para cada $r>0$ y $X \subseteq L$. Entonces,

$$
Y_{r}(X)=\arg \max _{Y \in L^{r}} W(Y \prec X) .
$$

La noción de $(r \mid p)$-centroide se introduce para definir la solución del líder cuando éste quiere instalar $p$ establecimientos de manera que la demanda total de los clientes que prefieren el conjunto de centros del seguidor sea mínima. Formalmente, un conjunto de localizaciones $X \in L^{p}$ es un $(r \mid p)$-centroide si y solamente si

$$
\max _{Y \in L^{r}} W(Y \prec X) \leq \max _{Y \in L^{r}} W(Y \prec Z), \quad \forall Z \in L^{p} .
$$

El valor de la puntuación de $X$ viene expresado por

$$
W_{r}^{*}(X)=\max _{Y \in L^{\prime}} W(Y \prec X)=W\left(Y^{*} \prec X\right), \quad \text { para } Y^{*} \in Y_{r}(X) .
$$

Entonces $X \in L^{p}$ es un $(r \mid p)$-centroide si

$$
W_{r}^{*}(X)=\min _{Z \in L^{p}} W_{r}^{*}(Z)
$$

Por lo tanto, un conjunto de localizaciones $X^{*} \in L^{p}$ es un conjunto ( $\left.r \mid p\right)$-centroide si y solamente si

$$
X^{*} \in \arg \min _{Z \in L^{p}} \max _{Y \in L^{r}} W(Y \prec Z) .
$$

Dado el conjunto de localizaciones $X$ para el líder, un $(r \mid X)$-medianoide es una solución óptima para el seguidor. Un $(r \mid p)$-centroide es una solución óptima para el líder.

\section{FORMULACIÓN LINEAL DEL PROBLEMA}

El problema del líder-seguidor con varios establecimientos se formula como un proceso de optimización en tres niveles simultáneos. Siguiendo la aproximación usada por Dobson y Karmakar (1987) y adaptada en Campos et al. (2010) tenemos:

- El problema de elección del cliente. Fijadas las localizaciones del líder y del seguidor, el cliente escoge el punto de servicio que prefiere.

- El problema de localización del seguidor. Fijadas las localizaciones del líder, el seguidor selecciona el conjunto de localizaciones de sus servicios que maximiza su demanda total captada.

- El problema de localización del líder. El líder determina el conjunto de localizaciones que minimiza la máxima demanda que puede capturar el seguidor. 
Para construir los programas lineales que describen estos tres problema enumeramos el conjunto de las localizaciones de los clientes $C=\left\{c_{k}\right.$ : $k \in[1 . . n]\}$ y el conjunto de posibles localizaciones de establecimientos $L=\left\{l_{i}\right.$ : $i \in[1 . . m]\}$, y denotamos $d_{k i}=d\left(c_{k}, l_{i}\right)$.

En el problema de elección de los clientes, las localizaciones del líder y del seguidor son conocidas. Por tanto, disponemos, $\forall i \in[1 . . m]$, de los datos:

$$
\bar{x}_{i}= \begin{cases}1 & \text { si el líder ha establecido un centro de servicio en } l_{i} \\ 0 & \text { en otro caso }\end{cases}
$$

$$
\bar{y}_{i}=\left\{\begin{array}{ll}
1 & \text { si el seguidor ha establecido un centro de servicio en } l_{i} \\
0 & \text { en otro caso }
\end{array} .\right.
$$

El problema de los clientes, en términos de las variables:

$$
z_{k i}= \begin{cases}1 & \text { si el cliente en } c_{k} \text { elige un centro de servicio en } l_{i} \\ 0 & \text { en otro caso }\end{cases}
$$

$\forall k \in[1 . . n], i \in[1 . . m]$, se formula de la manera siguiente:

$$
\begin{aligned}
& \sum_{i=1}^{m} z_{k i}=1, \\
& z_{k i} \leq \bar{x}_{i}+\bar{y}_{i}, \quad i \in[1 . . m] \\
& z_{k i} \leq \bar{y}_{i}+1-a_{j i}^{k} \bar{y}_{j}, \quad i, j \in[1 . . m] \\
& z_{k i} \leq \bar{y}_{i}+1-a_{j i}^{k} \bar{x}_{j}, \quad i, j \in[1 . . m] \\
& z_{k i} \leq \bar{x}_{i}+1-b_{j i}^{k} \bar{x}_{j}, \quad i, j \in[1 . . m] \\
& z_{k i} \leq \bar{x}_{i}+1-a_{j i}^{k} \bar{y}_{j}, \quad i, j \in[1 . . m] \\
& z_{k i} \in\{0,1\}, \quad i \in[1 . . m]
\end{aligned}
$$

donde $a_{i j}^{k} \mathrm{y} b_{i j}^{k}, \forall k \in[1 . . n], i, j \in[1 . . m]$, son parámetros dados por:

$$
a_{i j}^{k}=\left\{\begin{array}{lll}
1 & \text { si } & d_{k i}<d_{k j} \\
0 & \text { si } & d_{k i} \geq d_{k j}
\end{array} \text { y } b_{i j}^{k}=\left\{\begin{array}{lll}
1 & \text { si } & d_{k i} \leq d_{k j} \\
0 & \text { si } & d_{k i}>d_{k j}
\end{array} .\right.\right.
$$

En el problema del seguidor las localizaciones del líder son conocidas. Por tanto, $\forall i \in[1 . . m]$, disponemos de $\operatorname{los}$ datos $\bar{x}_{i}$ usados anteriormente $\mathrm{y}$ utilizamos las variables definidas, $\forall i \in[1 . . m]$, en la forma:

$$
y_{i}= \begin{cases}1 & \text { si el seguidor establece un centro de servicio en } l_{i} \\ 0 & \text { en otro caso }\end{cases}
$$


El problema del seguidor queda:

$$
\begin{array}{ll}
\text { Maximizar } & \sum_{i=1}^{m} \sum_{k=1}^{n} h_{k} z_{k i} \\
\text { Sujeto a: } & \sum_{i=1}^{m} y_{i}=r, \\
& \sum_{i=1}^{m} z_{k i} \leq 1, \quad k \in[1 . . n] \\
& z_{k i}-a_{i}^{k} y_{i} \leq 0, \quad i \in[1 . . m], k \in[1 . . n] \\
& z_{k i}, y_{i} \in\{0,1\}, \quad i \in[1 . . m], k \in[1 . . n]
\end{array}
$$

donde los coeficientes de la función objetivo son los parámetros $h_{k}=w\left(c_{k}\right)$, $k \in[1 . . n]$, y los coeficientes de la matriz de restricciones son los parámetros $a_{i}^{k}$ dados, $\forall k \in[1 . . n], i \in[1 . . m]$, por :

$$
a_{i}^{k}=\left\{\begin{array}{ll}
1 & \text { si } d_{k i}<\min \left\{d_{k j}: \bar{x}_{j}=1\right\} \\
0 & \text { en otro caso }
\end{array} .\right.
$$

Por tanto, éste es un problema de optimización con una función objetivo lineal, $m(n+1)$ variables binarias y $1+n(1+m)$ restricciones lineales.

Por último, para formular el problema del líder consideramos las variables:

$$
x_{i}= \begin{cases}1 & \text { si el líder establece un centro de servicio en } l_{i} \\ 0 & \text { en otro caso }\end{cases}
$$

donde $i \in[1 . . m]$, y las variables $z_{k i}^{j}, k \in[1 . . n], i \in[1 . . m], j \in J$, definidas por:

$$
z_{k i}^{j}= \begin{cases}1 & \text { si el cliente en } c_{k} \text { elige } l_{i} \text { frente a } j \in J \\ 0 & \text { en otro caso }\end{cases}
$$

siendo $J=\left[1 . .\left(\begin{array}{c}m \\ r\end{array}\right)\right]$. Es decir, $J$ es el conjunto de índices correspondientes a la enumeración de los elementos del conjunto de soluciones del seguidor, $L^{r}=\left\{Y_{j}\right.$ : $j \in J\}$. Introducimos los parámetros $c_{k i}^{j}$ dados por:

$$
c_{k i}^{j}= \begin{cases}1 & \text { si } d_{k i} \leq d\left(c_{k}, Y_{j}\right) \\ 0 & \text { en otro caso }\end{cases}
$$

para $k \in[1 . . n], i \in[1 . . m], j \in J$.

Entonces el problema del líder queda formulado de la manera siguiente: 


$$
\begin{aligned}
& \text { Minimizar } w \\
& \text { Sujeto a: } \sum_{i=1}^{m} x_{i}=p \text {, } \\
& \sum_{k=1}^{n} h_{k}-\sum_{i=1}^{m} \sum_{k=1}^{n} h_{k} z_{k i}^{j} \leq w, \quad j \in J \\
& \sum_{i=1}^{m} z_{k i}^{j} \leq 1, \quad k \in[1 . . n], j \in J \\
& z_{k i}^{j}-c_{k i}^{j} x_{i} \leq 0, \quad k \in[1 . . n], i \in[1 . . m], j \in J \\
& z_{k i}^{j}, x_{i} \in\{0,1\}, \quad k \in[1 . . n], i \in[1 . . m], j \in J \\
& w \geq 0 \text {. }
\end{aligned}
$$

Éste es un problema de optimización con una función objetivo lineal, $1+m+n m\left(\begin{array}{c}m \\ r\end{array}\right)$ variables binarias y $1+(1+m+n m)\left(\begin{array}{c}m \\ r\end{array}\right)$ restricciones lineales.

\section{EXTENSIONES DEL MODELO}

A partir del modelo básico, con restricciones únicamente en el número de localizaciones, podemos considerar diversas extensiones. Consideramos, 1) el modelo con umbral en la regla de elección y con restricciones en el número de localizaciones, 2) el modelo sin umbral en la regla de elección y con restricciones en el coste de las localizaciones, y 3) el modelo mixto con umbral en la regla de elección y con restricciones en el coste de las localizaciones.

\section{1) El modelo con umbral en la regla de elección y con restricciones en el número de localizaciones:}

Consideremos ahora que en el modelo básico descrito anteriormente para dos localizaciones distintas, $x$ e $y$ de $L$, la preferencia de cada cliente situado en $c$ se establece como una función monótona de la diferencia entre las distancias a ellas; es decir, como una función monótona de $d(c, y)-d(c, x)$ (ver Campos et al., 2011).

Consideramos que el establecimiento en $y \in L$ es preferido por el cliente en $c$ a $x \in L$ si $d(c, y)<d(c, x)-\delta$, y en caso contrario $x \in L$ es preferido a $y \in L$. Definidas de esta forma, las preferencias son asimétricas. Habría aversión al líder si $\delta<0$, y aversión al seguidor cuando $\delta \geq 0$. En el caso de una localizaciones $x$ con un centro del líder y otra localización $y$ con uno del seguidor en el que la diferencia de las distancias desde una ubicación con clientes $c$ a ellas coincide con el umbral, $d(c, x)-d(c, y)=\delta$, la regla favorece al líder al que se le asigna toda la demanda de los clientes en $c$. 
Para cada par de localizaciones $x, y \in L$, sea $C(y \prec x)$ el conjunto de localizaciones de clientes que cambian su elección de la localización $x$ por la localización $y$ más cercana. En vez de la expresión (1), en este modelo queda:

$$
C_{\delta}(y \prec x)=\{c \in C: d(c, y)<d(c, x)-\delta\} .
$$

Los clientes que cambian su elección al punto $y \in L$ desde un conjunto $X \subseteq L$ son aquellos más cercanos a $y$ que a cualquier punto de $X$ en una diferencia superior al umbral $\delta$.

El conjunto $C_{\delta}(Y \prec X)$ de localizaciones de clientes que captura el conjunto de localizaciones $Y \subseteq L$ frente al conjunto de localizaciones $X \subseteq L$ es el conjunto de puntos de $C$ para los que existe un punto de $Y$ más cerca que cualquier punto de $X$ en una diferencia superior al umbral $\delta$. Por tanto:

$$
\begin{aligned}
& C_{\delta}(Y \prec X)=\{c \in C: d(c, Y)<d(c, X)-\delta\}= \\
& =\{c \in C: \forall x \in X \exists y \in Y: d(c, y)<d(c, x)-\delta\} .
\end{aligned}
$$

La demanda capturada que debe maximizar el seguidor es:

$$
W_{\delta}(Y \prec X)=\sum\left\{w(c): c \in C_{\delta}(Y \prec X)\right\} .
$$

Por tanto, dados $r>0$ y $X \subseteq L$, el problema del seguidor es determinar el conjunto $Y^{*}$ tal que:

$$
W_{\delta}\left(Y^{*} \prec X\right)=\max \left\{W_{\delta}(Y \prec X): Y \in L^{r}\right\} .
$$

El valor o puntuación de cada solución $X$ del líder es:

$$
W_{r}^{*}(X)=\max _{Y \in L^{r}} W_{\delta}(Y \prec X) .
$$

El problema de optimización del líder es:

$$
\max _{Y \in L^{r}} W\left(Y \prec X^{*}\right)=\min _{X \in L^{p}} \max _{Y \in L^{r}} W(Y \prec X) .
$$

El problema de los clientes ahora es: 


$$
\begin{aligned}
& \sum_{i=1}^{m} z_{k i}=1, \quad k \in[1 . . n] \\
& z_{k i} \leq \bar{x}_{i}+\bar{y}_{i}, \quad i \in[1 . . m], k \in[1 . . n] \\
& z_{k i} \leq \bar{y}_{i}+1-a_{j i}^{k} \bar{y}_{j}, \quad i, j \in[1 . . m], k \in[1 . . n] \\
& z_{k i} \leq \bar{y}_{i}+1-c_{j i}^{k} \bar{x}_{j}, \quad i, j \in[1 . . m], k \in[1 . . n] \\
& z_{k i} \leq \bar{x}_{i}+1-b_{j i}^{k} \bar{x}_{j}, \quad i, j \in[1 . . m], k \in[1 . . n] \\
& z_{k i} \leq \bar{x}_{i}+1-c_{j i}^{k} \bar{y}_{j}, \quad i, j \in[1 . . m], k \in[1 . . n] \\
& z_{k i} \in\{0,1\}, \quad i \in[1 . . m], k \in[1 . . n]
\end{aligned}
$$

donde los parámetros $a_{i j}^{k}, b_{i j}^{k}$ y $c_{i j}^{k}, \forall k \in[1 . . n], i, j \in[1 . . m]$, vienen dados por:

$$
\begin{gathered}
a_{i j}^{k}=\left\{\begin{array}{lll}
1 & \text { si } & d_{k i}<d_{k j}-\delta \\
0 & \text { si } & d_{k i} \geq d_{k j}-\delta
\end{array}\right. \\
b_{i j}^{k}=\left\{\begin{array}{lll}
1 & \text { si } & d_{k i} \leq d_{k j}+\delta \\
0 & \text { si } & d_{k i}>d_{k j}+\delta
\end{array}\right. \\
c_{i j}^{k}=\left\{\begin{array}{lll}
1 & \text { si } & d_{k i}<d_{k j} \\
0 & \text { si } & d_{k i} \geq d_{k j}
\end{array}\right.
\end{gathered}
$$

Obsérvese que estamos considerando que si comparamos centros de la misma firma entonces el cliente prefiere el más próximo.

El problema del seguidor vuelve a ser (26):

$$
\begin{aligned}
& \text { Maximizar } \sum_{i=1}^{m} \sum_{k=1}^{n} h_{k} z_{k i} \\
& \text { Sujeto a: } \quad \sum_{i=1}^{m} y_{i}=r \text {, } \\
& \sum_{i=1}^{m} z_{k i} \leq 1, \quad k \in[1 . . n] \\
& z_{k i}-a_{i}^{k} y_{i} \leq 0, \quad i \in[1 . . m], k \in[1 . . n] \\
& z_{k i}, y_{i} \in\{0,1\}, \quad i \in[1 . . m], k \in[1 . . n]
\end{aligned}
$$

donde, ahora, los parámetros $a_{i}^{k}$ vienen dados, $\forall k \in[1 . . n], i \in[1 . . m]$, por :

$$
a_{i}^{k}=\left\{\begin{array}{ll}
1 & \text { si } d_{k i}<\min \left\{d_{k j}: \bar{x}_{j}=1\right\}-\delta . \\
0 & \text { en otro caso }
\end{array} .\right.
$$


Por último el problema del líder, sería, como en el modelo básico:

Minimizar $w$

$$
\begin{aligned}
& \text { Sujeto a: } \sum_{i=1}^{m} x_{i}=p \text {, } \\
& \sum_{k=1}^{n} h_{k}-\sum_{i=1}^{m} \sum_{k=1}^{n} h_{k} z_{k i}^{j} \leq w, \quad j \in J \\
& \sum_{i=1}^{m} z_{k i}^{j} \leq 1, \quad k \in[1 . . n], j \in J \\
& z_{k i}^{j}-c_{k i}^{j} x_{i} \leq 0, \quad k \in[1 . . n], i \in[1 . . m], j \in J \\
& z_{k i}^{j}, x_{i} \in\{0,1\}, \quad k \in[1 . . n], i \in[1 . . m], j \in J \\
& w \geq 0
\end{aligned}
$$

donde los parámetros $c_{k i}^{j}$ vienen dados, $\forall k \in[1 . . n], i \in[1 . . m], j \in J$, por:

$$
c_{k i}^{j}=\left\{\begin{array}{ll}
1 & \text { si } d_{k i} \leq d\left(c_{k}, Y_{j}\right)+\delta \\
0 & \text { en otro caso }
\end{array} .\right.
$$

\section{2) El modelo sin umbral en la regla de elección y con restricciones en el coste de las localizaciones:}

Por otro lado, si cada posible localización tiene asociado un coste y tanto el líder como el seguidor, en lugar de tener fijado el número de establecimientos que pueden abrir, tienen un presupuesto limitado, nos encontramos ante la segunda extensión propuesta que es el modelo sin umbral en la regla de elección y con restricciones en el coste de las localizaciones. Aunque en el modelo básico la limitación del número de centros que pueden abrirse lleva implícita una restricción presupuestaria, esta formulación implica costes iguales para todas las localizaciones. La segunda extensión del modelo permite considerar costes distintos para las posibles localizaciones de los establecimientos.

Sea $v$ una función de valor o coste sobre el conjunto de las posibles localizaciones del conjunto $L$, donde $v(x)$ representa el coste de la localización $x$. Sea $P$ el límite del coste total de los establecimientos que abrirá el líder y $R$ el de los que instalará el seguidor. Las condiciones $|X|=p$ y $|Y|=r$ son reemplazadas por $v(X) \leq P$ y $v(Y) \leq R$ respectivamente, siendo

$$
v(X)=\sum_{x \in X} v(x) \text { y } v(Y)=\sum_{y \in Y} v(y) .
$$

Sea $L^{Q}$ el conjunto de subconjuntos de $L$ con coste no superior a $Q$, es decir

$$
L^{Q}=\{X \subseteq L: v(X) \leq Q\} .
$$


En este modelo, el problema del cliente permanece exactamente igual que en el modelo básico dado en (23). Sin embargo, el problema del seguidor es, dados el presupuesto $R>0$ y las ubicaciones del líder $X \subseteq L$, determinar el conjunto $Y^{*} \in L^{R}$ tal que:

$$
W\left(Y^{*} \prec X\right)=\max \left\{W(Y \prec X): Y \in L^{R}\right\} .
$$

Y el problema del líder es, dados los presupuestos $P>0$ y $R>0$, determinar el conjunto $X^{*} \in L^{P}$ tal que:

$$
\max \left\{W\left(Y \prec X^{*}\right): Y \in L^{R}\right\}=\min \left\{\max \left\{W(Y \prec X): Y \in L^{R}\right\}: X \in L^{P}\right\} .
$$

El problema de programación lineal del seguidor queda:

$$
\begin{aligned}
\text { Maximizar } & \sum_{i=1}^{m} \sum_{k=1}^{n} h_{k} z_{k i} \\
\text { Sujeto a: } & \sum_{i=1}^{m} v_{i} y_{i} \leq R, \\
& \sum_{i=1}^{m} z_{k i} \leq 1, \quad k \in[1 . . n] \\
& z_{k i}-a_{i}^{k} y_{i} \leq 0, \quad i \in[1 . . m], k \in[1 . . n] \\
& z_{k i}, y_{i} \in\{0,1\}, \quad i \in[1 . . m], k \in[1 . . n] .
\end{aligned}
$$

Para este problema se usan los coeficientes $v_{i}=v\left(l_{i}\right), i \in[1 . . m]$, correspondientes a los costes de las posibles localizaciones de los establecimientos.

De forma análoga, el problema de programación lineal del líder queda:

Minimizar $w$

$$
\begin{aligned}
& \text { Sujeto a: } \sum_{i=1}^{m} v_{i} x_{i} \leq P, \\
& \sum_{k=1}^{n} h_{k}-\sum_{i=1}^{m} \sum_{k=1}^{n} h_{k} z_{k i}^{j} \leq w, \quad j \in J \\
& \sum_{i=1}^{m} z_{k i}^{j} \leq 1, \quad k \in[1 . . n], j \in J \\
& z_{k i}^{j}-c_{k i}^{j} x_{i} \leq 0, \quad k \in[1 . . n], i \in[1 . . m], j \in J \\
& z_{k i}^{j}, x_{i} \in\{0,1\}, \quad k \in[1 . . n], i \in[1 . . m], j \in J \\
& w \geq 0 \text {. }
\end{aligned}
$$

donde $j \in J$, es un índice que recorre todos los elementos de $L^{R}$, es decir, enu$\operatorname{meramos} L^{R}=\left\{Y_{j}: j \in J\right\}$. 


\section{3) El modelo mixto con umbral en la regla de elección y con restricciones en el coste de las localizaciones:}

Finalmente, consideramos el modelo mixto con umbral en la regla de elección y con restricciones en el coste de las localizaciones. Esta versión del modelo permite considerar costes no necesariamente iguales para las localizaciones candidatas al mismo tiempo que incorpora la posibilidad de reflejar distintos grados de aversión a los establecimientos del líder o del seguidor.

El conjunto de localizaciones de clientes que cambian su elección de una localización del líder en $X$ por una localización del seguidor en $Y$ más cercana es la función $C_{\delta}(Y \prec X)$ dada en (33) y a demanda capturada que debe maximizar el seguidor es $W_{\delta}(Y \prec X)$ dada en (34). Ahora bien, dados $R>0$ y $X \subseteq L$, el problema del seguidor es determinar el conjunto $Y^{*} \in L^{R}$ tal que:

$$
W_{\delta}\left(Y^{*} \prec X\right)=\max \left\{W_{\delta}(Y \prec X): Y \in L^{R}\right\} .
$$

Y el problema del líder es, dados los presupuestos $P>0$ y $R>0$, determinar el conjunto $X^{*} \in L^{P}$ tal que:

$$
\max \left\{W_{\delta}\left(Y \prec X^{*}\right): Y \in L^{R}\right\}=\min \left\{\max \left\{W_{\delta}(Y \prec X): Y \in L^{R}\right\}: X \in L^{P}\right\} .
$$

Por tanto, el problema de programación lineal de los clientes es el problema (38) con los parámetros dados en (39-41). El problema del seguidor es el problema (50) con los parámetros dados en (43) y el del líder es (51) con los parámetros dados en (45).

\section{EJEMPLO}

Ilustramos ahora mediante varios ejemplos ad-hoc la aplicación de los modelos propuestos. Utilizamos casos sencillos con un número suficientemente pequeño de variables y restricciones que permite seguir la explicación. Formulamos los ejemplos sobre el grafo de 11 vértices representado en la Figura 1. El número próximo al centro de cada arista corresponde a su longitud, y el número en cada cuadrado corresponde al volumen de la demanda de los usuarios ubicados en el vértice indicado junto al cuadrado. Suponemos que el conjunto de localizaciones posibles $L$ está constituido por todos los vértices de este grafo.

Para el modelo básico tomando un número $p=3$ de localizaciones del líder y un número $r=2$ de localizaciones para el seguidor. La mejor solución para el líder es $X^{*}=\left\{v_{6}, v_{8}, v_{10}\right\}$, a la que corresponde la elección del seguidor $Y^{*}=\left\{v_{9}\right.$, $\left.v_{11}\right\}$. Entonces el líder conseguiría una demanda de $40 \mathrm{y}$ el seguidor de 20.

Si introducimos un umbral $\delta=5$ para la diferencia de distancia a los competidores, la mejor solución para el líder es $X^{*}=\left\{v_{6}, v_{8}, v_{11}\right\}$ y la mejor elección del seguidor es $Y^{*}=\left\{v_{1}, v_{9}\right\}$. El líder conseguiría un peso de 42 y el seguidor 18 . 
Figura 1

Grafo del Ejemplo

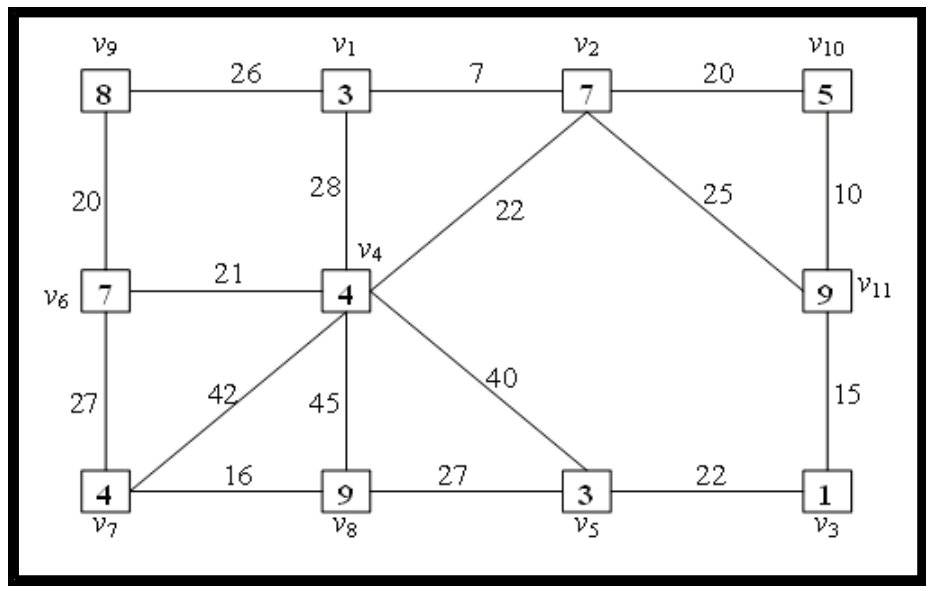

Fuente: Elaboración propia.

Supongamos ahora que los costes de las localizaciones en los 11 vértices vienen dados por el vector $c=[5,6,7,5,6,7,6,6,5,5,5]$ pero no consideramos umbral para las distancias. Si el líder tiene un presupuesto de 15 unidades monetarias y el seguidor tiene un presupuesto de 12 tenemos un ejemplo del modelo extendido con la introducción de costes de establecimiento. Para este modelo la mejor solución para el líder es $X^{*}=\left\{v_{6}, v_{10}\right\}$ y el seguidor responde con la selección $Y^{*}=\left\{v_{8}, v_{11}\right\}$. El líder conseguiría un peso de 34 con un coste de 12 unidades monetarias y el seguidor conseguiría 26 con un coste de 11 unidades.

Finalmente, si con estos mismos costes de establecimiento anteriores consideramos además el umbral $\delta=3$ para la diferencia de distancias, tenemos el modelo mixto con la doble extensión propuesta en este trabajo. En este caso, la mejor solución para el líder es $X^{*}=\left\{v_{1}, v_{4}, v_{11}\right\}$ mientras que el seguidor responde con la selección $Y^{*}=\left\{v_{8}, v_{9}\right\}$. El líder conseguiría ahora un peso de 36 con un coste de 15 unidades monetarias y el seguidor conseguiría 24 con un coste de 11 unidades.

\section{CONCLUSIONES}

En este trabajo consideramos la formulación matemática y solución del problema de localización competitiva del líder-seguidor para bienes esenciales. Partiendo del modelo básico discreto con una regla de elección binaria orientada al líder consideramos dos líneas en las que extender el modelo hacia situaciones más realistas y versátiles. Por un lado, consideramos la existencia de un umbral 
en las diferencias de distancias para que los clientes manifiesten su preferencia y por otro lado contemplamos la existencia de diferencias en los costes localización. Partiendo de la formulación como programa lineal del problema básico concebido como una terna de problemas de optimización simultáneos, el problema del cliente, el problema del seguidor y el problema del líder, obtenemos formulaciones lineales paralelas a las obtenidas para el modelo básico.

El trabajo realizado sugiere varias líneas de investigación futura. Por un lado tenemos la modificación de la regla de elección del cliente, con el objetivo de representar el comportamiento del consumidor de forma más realista. Aunque por su simplicidad y ventajas metodológicas hemos utilizado la regla de elección binaria, este modelo presenta ciertas deficiencias respecto a su capacidad de representación de comportamientos reales. Es preciso contemplar formas alternativas de resolver los empates donde resultan funciones de elección discontinuas. Por otra parte podemos considerar también la aplicación de estas ideas al caso de bienes no esenciales. Otra línea de estudio tiene que ver con la explotación de las formulaciones lineales propuestas para diseñar algoritmos de resolución del problema del líder. Las formulaciones lineales obtenidas para el problema del líder en las distintas extensiones son de un tamaño tal que no permite su aplicación directa para obtener una solución por un software de programación lineal estándar. Por tanto, se hace necesario utilizar estas formulaciones para idear procedimientos de solución eficientes aprovechando técnicas metaheurísticas y otras técnicas más elaboradas de programación matemática.

\section{REFERENCIAS BIBLIOGRÁFICAS}

ALEKSEEVA, E.; KOCHETOVA, N.; KOCHETOV, Y. y PLYASUNOV, A. (2010). "Heuristic and exact methods for the discrete $(r \mid p)$-centroid problem". En Cowling, P.I. y Merz, P. (ed.): EvoCOP'10 Proceedings of the $10^{\text {th }}$ European Conference on Evolutionary Computation in Combinatorial Optimization (pp.11-22). Berlin: Springer-Verlag.

BENATI, S. y LAPORTE, G. (1994). "Tabu search algorithms for the $\left(r \mid X_{p}\right)$-medianoid and $(r \mid p)$-centroid problems". Location Science, 2, pp. 193-204.

BERMAN, O. y KRASS D. (2002). "The generalized maximal covering location problem". Computers \& Operations Research, 29, pp. 563-581.

BHADURY, J.; EISELT, H.A. y JARAMILLO, J.H. (2003). "An alternating heuristic for medianoid and centroid problems in the plane". Computers \& Operations Research, 30, pp. 553-565. 
CAMPOS, C.; SANTOS-PEÑATE D.R. y MORENO J.A. (2010). "An exact procedure and LP formulations for the leader-follower problema". TOP, 18(1), pp. 97-121.

CAMPOS, C.; SANTOS-PEÑATE, D.R. y MORENO, J.A. (2011). "Competencia espacial por cuotas de mercado: el problema del líder-seguidor mediante programación lineal”. Rect@. Revista Electrónica de Comunicaciones y Trabajos de ASEPUMA,12, pp. 68-84.

DASKIN, M.S. (1995). Network and Discrete Location: Models, Algorithms, and Applications. New York: Wiley.

DEVLETOGLOU, N.E. (1965). "A dissenting view of duopoly and spatial competition". Economica, pp. 141-160.

DEVLETOGLOU, N.E. y DEMETRIOU, P.A. (1967). "Choice and threshold: a further experiment in spatial duopoly". Economica, pp. 351-371.

DOBSON, G. y KARMARKAR, U.S. (1987). "Competitive location on a network". Oper. Res., 35, pp. 565-574.

EISELT, H.A. y LAPORTE, G. (1989). “Competitive spatial models”. European Journal of Operational Research, 39, pp. 231-242.

EISELT, H.A. y LAPORTE, G. (1996). "Sequential location problems". European Journal of Operational Research, 96, pp. 217-231.

EISELT, H.A.; LAPORTE, G. y THISSE, J.F. (1993). "Competitive spatial models: A framework and bibliography". Transportation Science, 27(1), pp. 44-54.

FRIESZ, T.L.; MILLER, T. y TOBIN, R.L. (1988). "Competitive network facility location models: a survey". Papers of the Regional Science Association, 65, pp. 47-57.

GANDHI, R.; KHULLER, S. y SRINIVASAN, A. (2004). "Approximation algorithms for partial covering problems". Journal of Algorithms, 53(1), pp. 5584.

HAKIMI, S.L. (1983). "On locating new facilities in a competitive environment". European Journal of Operational Research, 12, pp. 29-35.

HAKIMI, S.L. (1990). "Location with spatial interactions: competitive locations and games". En Mirchandani, P.B. y Francis, R.L. (ed.): Discrete Location Theory (pp. 439-478). New York: Wiley.

KRESS, D. y PESCH, E. (2012). "Sequential competitive location on networks". European Journal of Operational Research, 217, pp. 483-499.

PLASTRIA, F. (1990). "Static competitive facility location: an overview of optimization approaches". European Journal of Operational Research, 129, pp. 461-470.

REDONDO, J.L.; FERNÁNDEZ, J.; GARCÍA, I. y ORTIGOSA, P.M. (2010). "Heuristics for the facility location and design (1|1)-centroid problem on the plane". Computational Optimization and Applications, 45(1), pp. 111-141. 
REVELLE C. (1986). "The maximum capture or sphere of influence location problem: Hotelling revisited on a network". Journal of Regional Science, 26(2), pp. 343-358.

SANTOS-PEÑATE, D.R.; SUÁREZ-VEGA, R. y DORTA-GONZÁLEZ, P. (2007). "The leader-follower location model". Networks and Spatial Economics, 7, pp. 45-61.

SERRA, D. y REVELLE, C. (1994). "Market capture by two competitors: the preemptive location problem". Journal of Regional Science, 34(4), pp. 549561.

SERRA, D. y REVELLE, C. (1995). "Competitive location in discrete space". En Z. Drezner (ed.): Facility location: A survey of applications and methods (pp. 367-386). Berlin: Springer.

SPOERHASE, J. y WIRTH, H.C. (2009). "(r|p)-centroid problems on paths and trees". Theoretical Computer Science, 410(47-49), pp. 5128-5137.

SANTOS-PEÑATE, D.R.; SUÁREZ-VEGA, R. y DORTA-GONZÁLEZ, P. (1999). "Equilibrio localización-atractivo para dos competidores con cadenas de centros de servicio igualmente atractivos". Estudios de Economía Aplicada, 12, pp. 145-164.

SUÁREZ-VEGA, R.; SANTOS-PEÑATE, D.R. y DORTA-GONZÁLEZ, P. (2004). "Competitive multifacility location on networks: the (r|Xp)-medianoid problem". Journal of Regional Science, 44(3), pp. 569-588. 